Pacific

Journal of

Mathematics

HOMOLOGICALLY TRIVIAL ACTIONS ON CYCLIC COVERINGS OF KNOTS

Luisa Paoluzzi and Marco Reni

Volume 188 No. 1

March 1999 


\title{
HOMOLOGICALLY TRIVIAL ACTIONS ON CYCLIC COVERINGS OF KNOTS
}

\author{
Luisa PaOluzzi and Marco Reni
}

\begin{abstract}
A classical theorem of Hurewitz says that the isometry group of a closed 2-dimensional hyperbolic manifold acts faithfully on its first homology group. The analogous theorem in dimension 3 is false. In this paper we consider the class of 3-manifolds which are cyclic branched coverings of knots in the 3-sphere $S^{3}$. We characterize the isometry group actions which are homologically faithful in the case of $p$-fold cyclic coverings of knots when $p$ is sufficiently large. This characterization is given in terms of the knot polynomials.
\end{abstract}

\section{Introduction.}

A classical theorem of Hurewitz says that the isometry group of a closed 2dimensional hyperbolic manifold acts faithfully on its first homology group. The analogous theorem in dimension 3 is false. In this paper we consider the class of 3-manifolds which are cyclic branched coverings of knots in the 3 -sphere $\mathbf{S}^{3}$. We give necessary and sufficient conditions, in terms of the knot polynomials, for the effectiveness of the isometry group action on the first homology group in the case of hyperbolic manifolds which are $p$-fold cyclic coverings of knots when $p$ is sufficiently large.

The special properties of homologically trivial actions on a CW-complex have been studied by mathematicians since the 30's with the pioneering work of Paul Smith. The technique of Smith's exact sequences has later been generalized by Borel in his Princeton seminar. In Section 3 we shall specialize to the 3-dimensional case some of these classical results. More generally homologically trivial actions on manifolds can be addressed by the methods of the general theory of transformation groups. However the point of this paper is that the 3 -dimensional case has some special features which are worth to be written.

The most elementary class of homologically trivial actions on hyperbolic 3-manifolds are finite group actions on homology 3-spheres (see [27] and references therein). More interestingly, one can use Dehn surgery on knots to construct homologically trivial actions on hyperbolic 3-manifolds which 
are not homology 3-spheres: We will briefly discuss this construction in Section 2 and Section 4. In this paper we are mainly interested in the class of 3 -manifolds which are cyclic coverings of knots in $\mathbf{S}^{3}$. The starting point of our work are the classical homology invariants which have been used to classify knots since the beginning of knot theory. The most popular among these invariants is the Alexander polynomial and its application is often successful to distinguish knots with few crossings and find their periods. More recently, mathematicians have turned their attention to other kinds of knot polynomials to deal with those cases where abelian invariants do not work. However the classical homology invariants seem to be more appropriate to our purposes.

We will often make use of the following two facts which are well-known:

i) The Alexander polynomial of a periodic knot (see Section 2 for precise definitions and statements) satisfies the so-called Murasugi conditions [21]. These conditions can be explicitly computed and have been used to test periods of knots [4]. It has been conjectured and proved in some cases [8] that Murasugi conditions for a knot polynomial $\Delta$ are also sufficient to find a periodic knot with Alexander polynomial $\Delta$. Further work on periodicity conditions on Alexander polynomials can be found in [22], [23], [29].

ii) If $M$ is a $p$-fold cyclic branched covering of a knot in $\mathbf{S}^{3}$, then the first homology group of $M$ has some well-known properties. For example, if $p$ is prime then $H_{1}\left(M, \mathbb{Z}_{p}\right)=0$ [12]. If $p$ is odd and prime, Plans [24] has shown that $H_{1}(M, \mathbb{Z})$ is a direct double isomorphic as an abelian group to $G \oplus G$ (see also $[\mathbf{9}],[\mathbf{1 4}]$ ). There have been also various attempts of classifying algebraically the class of groups which occur as homology groups of cyclic coverings of knots [5] and this classification has finally been completed in [6] and [7]. See also [11] for periodicity phenomena in the homology groups of cyclic coverings of knots.

Our main Theorems are the following:

Theorem 5. Let $M$ be a p-fold cyclic branched covering of a knot $K$ in $\mathbf{S}^{3}$ for some $p$ prime. Suppose that:

i) $M$ is hyperbolic and not a homology 3-sphere.

ii) $K$ has neither cyclic nor free periods.

There exists a number $N$ depending on $K$ such that if $p>N$, then the orientation-preserving isometry group of $M$ acts faithfully on the first homology group of $M$.

We make a brief comment about the number $N$ which appears in the statement of the Theorem. As it will be explained later in this introduction, if the order of the covering is sufficiently high, it follows from Thurston's theory that the isometry group of $M$ consists of the set of lifts of the isometries of $K$. The constant $N$ for which this phenomenon occurs is not universal 
and depends on the knot $K$. It is possible [28] to give a numerical estimate of $N$ in terms of geometric quantities related to the knot.

Theorem 6. Let $M$ be a p-fold cyclic branched covering of a knot $K$ in $\mathbf{S}^{3}$ for some $p$ prime. Denote by $q_{1}, \ldots, q_{s}$ the prime cyclic periods of $K$ and by $K_{1}, \ldots, K_{s}$ their fixed-point sets in $\mathbf{S}^{3}$. Suppose that:

i) $M$ is hyperbolic and not a homology 3-sphere.

ii) $K$ has no free periods.

iii) The linking number $l k\left(K, K_{i}\right)(i=1, \ldots, s)$ is 1 .

There exists a number $N$ depending on $K$ such that if $p>N$, then the orientation-preserving isometry group of $M$ acts faithfully on the first homology group of $M$ if and only if the knot $K$ does not satisfy the $\mathcal{M}$-condition for the primes $q_{1}, \ldots, q_{s}$.

Theorem 6 is a special case of a more general theorem (Theorem 4) which is proved in Section 4. Despite the long statement, the hypotheses of Theorem 6 are quite general. We make now several comments to explain these hypotheses.

- The $\mathcal{M}$-condition appearing in the statement of Theorem 6 comes from the Murasugi conditions and we postpone its precise definition to Section 2: It is a computable condition on a knot polynomial.

- It is possible to work out a corresponding $\mathcal{M}$-condition also in the case that $\mathrm{lk}\left(K, K_{i}\right)$ is not 1 . However this condition is more involved and algebraically complicated. Moreover, in the case $\operatorname{lk}\left(K, K_{i}\right)=1$, we are able to provide concrete examples of knots satisfying the $\mathcal{M}$-condition.

- The hypothesis ii) that $K$ has no free periods is not restrictive, since we shall prove in Section 4 (Theorem 3) that given a 3-manifold which is the cyclic covering of a knot with a free period, it comes down to the case of a cyclic covering of a knot with a cyclic period (we postpone precise definitions to Section 2).

- The only essential condition in Theorem 6 is that the order $p$ of the covering is sufficiently large. This is in fact the limit of the present investigation. The problem we are faced with, is that if a hyperbolic 3-manifold $M$ is presented as the cyclic covering of a knot $K$, then its isometry group is not known a priori by simply looking at the knot itself. In fact every isometry of $K$ lifts to an isometry of $M$, but, in general, there exist isometries of $M$ which do not descend to $K$. The isometries which do not descend to $K$ are said hidden to $K$. Now, if the order of the covering is sufficiently high, it follows from Thurston's theory $([\mathbf{1 7}],[\mathbf{2 6}],[\mathbf{2 8}])$ that hidden isometries do not exist and we can solve the problem of the existence of homologically trivial actions on $M$, by only looking at the knot and its homology invariants. However, for low order coverings, hidden isometries, in general, do exist [28] and the structure of the isometry group is only partially understood [26]. This 
phenomenon is investigated in more detail in Section 8 where we prove also a partial result for the case of actions by hidden isometries.

The paper is organized as follows:

In Section 2 we give some examples of homologically trivial actions on 3manifolds and state the $\mathcal{M}$-condition. In Section 3 we discuss some classical theorems of transformation group theory for the 3-dimensional case. In Section 4 we prove our more general theorem. In Sections 5 and 6 we prove Theorem 5 and 6 . In Section 7 we discuss some examples of knots which satify the $\mathcal{M}$-condition. In Section 8 we discuss the phenomenon of hidden isometries.

\section{Preliminaries and statement of $\mathcal{M}$-condition.}

A knot $K$ in $\mathbf{S}^{3}$ is said to have cyclic period $n$ if there is a diffeomorphism $f$ of the pair $\left(\mathbf{S}^{3}, K\right)$ of order $n$ which preserves the orientation of both $K$ and $\mathbf{S}^{3}$ with non-empty fixed-point set a circle disjoint from $K$. By the positive solution of the Smith conjecture [20] the fixed-point set of such a diffeomorphism is unknotted and the diffeomorphism is equivalent to a rotation around its fixed-point set. A knot $K$ is said to have free period $n$ if there is a diffeomorphism of the pair $\left(\mathbf{S}^{3}, K\right)$ of order $n$ which preserves the orientation of both $K$ and $\mathbf{S}^{3}$ with empty fixed-point set.

If $K$ is a hyperbolic knot, that is its complement admits a complete Riemannian structure with constant negative curvature, as a consequence of Mostow's Rigidity Theorem, any periodic diffeomorphism of $\left(\mathbf{S}^{3}, K\right)$ can be chosen to be an isometry on $\mathbf{S}^{3}-K$. More generally, if $M$ is a hyperbolic 3-manifold with finite volume, then the mapping class group of $M$ (which is called the symmetry group of $M$ ) is finite and isomorphic to the isometry group of $M$. We shall be concerned only with the orientation-preserving symmetry group of $K$, that is with free or cyclic periods or invertibility (a hyperbolic knot is invertible if there is an involution of $\left(\mathbf{S}^{3}, K\right)$ preserving the orientation of $\mathbf{S}^{3}$ with non-empty fixed-point set a circle intersecting $K$ into two points). Thus the orientation-preserving symmetry group of $K$ is isomorphic to a dihedral group $\mathbf{D}_{n}$ of order $2 n$ or the cyclic group $\mathbb{Z}_{n}$ according if $K$ is invertible or not.

It is quite easy to construct homologically trivial actions on hyperbolic 3-manifolds by using Dehn surgery on a knot in $\mathbf{S}^{3}$.

Recall the process of Dehn surgery. Let $K \subset \mathbf{S}^{3}$ be a knot, $T$ a regular neighbourhood of $K$ and $M$ the closed complement of $T$. On $\partial T=\partial M$ (a 2-torus) we have two distinguished simple closed curves: A meridian $m$ which bounds a disk in $T$ and generates $H_{1}(M)$; a longitude $l$ which is trivial in $H_{1}(M)$. Let $V$ be a full torus with meridian $\bar{m}$ bounding a disk in $V$. Identify $\partial M$ with $\partial V$ by a homeomorphism $h$ mapping $\bar{m}$ to $m^{p} l^{q}$ for 
$p, q \in \mathbb{Z}$ and $\operatorname{gcd}(p, q)=1$. The closed orientable 3 -manifold

$$
M_{p, q}:=M \cup_{h} V
$$

is said to be obtained by $p / q$-surgery on $K$.

Let now $K \subset \mathbf{S}^{3}$ be a knot with period $n>1$ and $f$ the diffeomorphism of the pair $\left(\mathbf{S}^{3}, K\right)$ of order $n$. The map $f$ acts homologically trivially on $M$ and, since it is orientation-preserving, then [16, Lemma 5.2] it extends to an orientation-preserving periodic map, say $f_{p, q}$ of $M_{p, q}$ for any pair $(p, q)$. The map $f_{p, q}$ fixes setwise the core of the added solid torus and has the same period $n$ has $f$ (because any isotopy of $M_{p, q}$ fixing setwise the core of the added solid torus restricts to an isotopy of $\left.S^{3}-K\right)$. Note that

$$
H_{1}\left(M_{p, q}\right) \cong H_{1}(M) /<m^{p} l^{q}>\cong \mathbb{Z}_{p}
$$

In particular $H_{1}\left(M_{p, q}\right)$ is generated by the image of the curve $m$ in $M_{p, q}$ : Therefore $f_{p, q}$ acts homologically trivially on $M_{p, q}$. This construction can be made hyperbolic: If $K$ is hyperbolic, then, by Thurston's Hyperbolic Dehn Surgery Theorem $([\mathbf{3 1}]$ and $[\mathbf{2 5}$, Section 10.5]), for all but finitely many $(p, q)$, the manifold $M_{p, q}$ is also hyperbolic.

Another application of the Dehn surgery construction above will be described at the beginning of Section 4. The case of manifolds which are presented as cyclic branched covering of knots is much more difficult and it is the subject of the rest of the paper. Let now $M$ be a cyclic $p$-fold branched covering of a knot $K$ in $\mathbf{S}^{3}$ and denote by $\Delta$ the Alexander polynomial of $K$. A classical result of Fox [10] says that

$$
\text { order } H_{1}(M)=\left|\prod_{i=0}^{p-1} \Delta\left(\rho^{i}\right)\right|
$$

where $\rho$ is a primitive $p$ th root of unity. The convention in the formula above is that the order of an infinite group is 0 . Thus $H_{1}(M)$ has infinite order if and only if some root of $\Delta$ is a primitive $p$ th root of unity.

Let us now state the $\mathcal{M}$-condition for a periodic knot. Let $K$ be a knot with cyclic period $q$ and $g$ a cyclic diffeomorphism of $\left(\mathbf{S}^{3}, K\right)$ of order $q$. Denote by $K^{\prime}$ the fixed-point set of $g$ and by $G$ the group generated by $g$. As we have seen above the topological space underlying the quotient $\mathbf{S}^{3} / G$ is homeomorphic to $\mathbf{S}^{3}$; let $\bar{K}$ and $\bar{K}^{\prime}$ be the projections of $K$ and $K^{\prime}$ to this quotient. Denote by $\Delta, \bar{\Delta}$ and $\Delta_{L}$ the Alexander polynomials of $K, \bar{K}$ and $L:=\bar{K} \cup \bar{K}^{\prime}$. Let $\lambda:=\operatorname{lk}\left(\bar{K}, \bar{K}^{\prime}\right)$ be the linking number of $\bar{K}$ and $\bar{K}^{\prime}$. 
Define $\rho_{\lambda}(t):=\sum_{i=0}^{\lambda-1} t^{i}$. The following relations are proved in [21]:

a)

$$
\rho_{\lambda}(t) \Delta(t)=\prod_{j=0}^{q-1} \Delta_{L}\left(t, \theta^{j}\right)
$$

b) $\quad \rho_{\lambda}(t) \bar{\Delta}(t)=\Delta_{L}(t, 1)$

where equalities are thought up to invertible elements in the ring of Laurent polynomials with coefficients in $\mathbb{Z}[\theta]$, respectively $\mathbb{Z}$, and $\theta$ is a primitive $q$ th-root of unity. In the case $\lambda=1$, which we shall be mainly concerned with, the relations above assume the simplified form:

$$
\text { a) } \begin{aligned}
\Delta(t) & =\prod_{j=0}^{q-1} \Delta_{L}\left(t, \theta^{j}\right) \\
\text { b) } \bar{\Delta}(t) & =\Delta_{L}(t, 1) .
\end{aligned}
$$

By applying Fox's formula to $\Delta$ and $\bar{\Delta}$ we have that the orders of the first homology groups of the $p$-fold cyclic branched coverings $M$ and $\bar{M}$ of $K$, respectively $\bar{K}$, are:

$$
\begin{aligned}
& \text { order } H_{1}(M)=\left|\prod_{i=0}^{p-1} \prod_{j=0}^{q-1} \Delta_{L}\left(\rho^{i}, \theta^{j}\right)\right| \\
& \text { order } H_{1}(\bar{M})=\left|\prod_{i=0}^{p-1} \Delta_{L}\left(\rho^{i}, 1\right)\right| .
\end{aligned}
$$

We shall say that a knot $K$ of cyclic period $q$ satisfies the $\mathcal{M}$-condition for the number $p$ if

$$
\text { order } H_{1}(\bar{M})=\text { order } H_{1}(M)
$$

that is if and only if:

$$
\mathcal{M} \text { - condition: }\left|\prod_{i=0}^{p-1} \prod_{j=1}^{q-1} \Delta_{L}\left(\rho^{i}, \theta^{j}\right)\right|=1 .
$$

The last item of this Section is the statement of the $\mathcal{M M}$-condition, which we shall need only for the more general Theorem 4 , but not for the special cases of Theorem 5 and 6 . The generalization of the Fox's formula above to the case of coverings of links with more than one component is not straightforward and has been done by Mayberry and Murasugi [18]. Before discussing this generalization, we briefly explain what we mean by cyclic coverings of links with more than one component, because there are in the literature different definitions of the same concept. Let $L$ be a link in $\mathbf{S}^{3}$ with $\nu$ components $K_{1}, \ldots, K_{\nu}$. Each epimorphism $\psi: \pi_{1}\left(\mathbf{S}^{3}-L\right) \longrightarrow \mathbb{Z}_{n}$ factors through $H_{1}\left(\mathbf{S}^{3}-L\right)$ which is a free abelian group on $\nu$ generators. These generators can be chosen to be $\nu$ meridianal curves $m_{1}, \ldots, m_{\nu}$ around 
$K_{1}, \ldots, K_{\nu}$. Each epimorphism $\psi$ defines a $n$-fold unbranched covering of $\mathbf{S}^{3}-L$ and a $n$-fold branched covering of $L$ obtained by glueing some solid tori to $\mathbf{S}^{3}-L$ along its boundary components. We say that the branched covering is a cyclic branched covering of $L$ if each $m_{i}$ is mapped to a generator of $\mathbb{Z}_{n}$ (this is always the case if $n$ is a prime number). A particular special case, which we call strictly-cyclic is that all $m_{i}$ are mapped to the same generator of $\mathbb{Z}_{n}$ : These coverings have first been considered by Hosokawa $[15]$.

We can now state the $\mathcal{M M}$-condition for a knot. We first recall the notation above. Let $K$ be a knot with cyclic period $p$ and $g$ a cyclic diffeomorphism of $\left(\mathbf{S}^{3}, K\right)$ of order $p$. Denote by $K^{\prime}$ the fixed-point set of $g$ and by $G$ the group generated by $g$. The topological space underlying the quotient $\mathbf{S}^{3} / G$ is homeomorphic to $\mathbf{S}^{3}$; let $\bar{K}$ and $\bar{K}^{\prime}$ be the projections of $K$ and $K^{\prime}$ to this quotient. Denote by $\Delta, \bar{\Delta}, \bar{\Delta}^{\prime}$ and $\Delta_{L}$ the Alexander polynomials of $K, \bar{K}, \bar{K}^{\prime}$ and $L=\bar{K} \cup \bar{K}^{\prime}$. Let $\psi: H_{1}\left(\mathbf{S}^{3}-L\right) \longrightarrow \mathbb{Z}_{p}$ an epimorphism such that, for some fixed meridians $m$ and $m^{\prime}$ around $\bar{K}$ and $\bar{K}^{\prime}$ we have $\psi(m)=h$ and $\psi\left(m^{\prime}\right)=h^{r+1}$ where $h$ is a generator of $\mathbb{Z}_{p}$ and $r$ a positive integer $0 \leq r \leq p-2$. Denote by $Q$ the $p$-fold cyclic branched covering of $L$ corresponding to $\psi$. Define the modified Alexander polynomial $\tilde{\Delta}_{L}$ of $L$ as $\tilde{\Delta}_{L}(s, t)=\Delta_{L}(s, t)$ if $s \neq 1$ and $t \neq 1 ; \tilde{\Delta}_{L}(1, t)=\bar{\Delta}^{\prime}(t)$ and $\tilde{\Delta}_{L}(s, 1)=\bar{\Delta}(s)$. Then Mayberry and Murasugi showed [18, Corollary 4.5.1 and Theorem 10.1] that:

$$
\text { order } H_{1}(Q)=p\left|\prod_{i=0}^{p-1} \tilde{\Delta}_{L}\left(\rho^{i}, \rho^{i+r}\right)\right|
$$

where $\rho$ is a primitive $p$ th root of unity. We know (see above) that the order of the first homology group of the $p$-fold cyclic branched covering of $M$ is

$$
\text { order } H_{1}(M)=\left|\prod_{i=0}^{p-1} \prod_{j=0}^{p-1} \Delta_{L}\left(\rho^{i}, \theta^{j}\right)\right| \text {. }
$$

The $\mathcal{M M}$-condition for a knot of period $p$ is that

$$
\text { order } H_{1}(Q)=p \text { order } H_{1}(M)
$$

that is:

$$
\mathcal{M M} \text { - condition: }\left|\prod_{i=0}^{p-1} \prod_{j=0}^{p-1} \Delta_{L}\left(\rho^{i}, \theta^{j}\right)\right|=\left|\prod_{i=0}^{p-1} \tilde{\Delta}_{L}\left(\rho^{i}, \rho^{i+r}\right)\right|
$$

for at least one value of $r$ with $0 \leq r \leq p-2$. 


\section{Homologically trivial actions on 3-manifolds.}

In this Section we apply transfer arguments and Smith's exact sequences to the case of cyclic groups acting on a 3-manifold. One has to distinguish carefully between the case when a group acts freely and the case with nonempty fixed-point set.

We first fix some common notation. Let $M$ be a compact 3-manifold on which a finite group $G$ acts smoothly and orientation-preservingly. Denote by $M / G$ the quotient orbifold and by $M_{G}$ the underlying topological space. Note that if $G$ acts freely then $M / G=M_{G}$. In the following it will be important to distinguish between $M / G$ and $M_{G}$ and between their fundamental groups, so we briefly recall this notion.

To define the fundamental group of an orbifold, one constructs the universal covering orbifold in the same way as one constructs universal covering spaces and the same uniqueness holds. Recall that a continuous map $f: X \longrightarrow Y$ is a covering if any point $y \in Y$ has a neighbourhood $U$ such that $f^{-1} U$ is the disjoint union of sets $V_{\lambda}$ for $\lambda \in \Lambda$ such that $f \mid V_{\lambda} \longrightarrow U$ is a homeomorphism. If $X$ and $Y$ are orbifolds and $f: X \longrightarrow Y$ is an orbifold map, one defines an orbifold covering in the same way except that one allows $f \mid: V_{\lambda} \longrightarrow U$ to be the natural quotient map between two quotients of $\mathbb{R}^{n}$ by finite groups, one of which is a subgroup of the other. There are general arguments for showing that any orbifold has a universal covering orbifold. As with coverings in the ordinary sense, the orbifold is the quotient of this universal covering by the action of a group, which is called the orbifold fundamental group of the orbifold. For more information about this topic see [25, Chapter 13].

We denote by $H_{1}\left(M_{G}\right)$ the homology of the quotient space $M_{G}$ and by $H_{1}(M / G)$ the abelianized of the orbifold fundamental group of $M / G$. Again if $G$ acts freely the two groups coincide.

We first prove the following:

Theorem 1. Let $M$ be a compact 3-manifold and $G$ a finite cyclic group acting smoothly on $M$. Then $G$ acts homologically trivially on $M$ if and only if $H_{1}(M / G)$ is an extension of a normal subgroup $H \cong H_{1}(M)$ by a cyclic subgroup $F \cong G$.

We remark that Theorem 1 can be obtained by a standard application of a Cartan-Leray spectral sequence [3]. We prefer however to exhibit an elementary proof of this result which makes use only of group theory. In the proof of this Theorem it is not essential that we are dealing with manifolds of dimension 3 .

Lemma 1. Let $M$ be a compact 3-manifold and $G$ a finite group acting smoothly on $M$. Denote by $H_{1}(M)^{G}$ the subgroup of elements of $H_{1}(M)$ 
which are fixed by $G$ for the induced action of $G$ on the first homology group of $M$. Let $\pi$ be the set of prime numbers which divide $|G|$ and $\pi^{\prime}$ the set of prime numbers not contained in $\pi$. We have $H_{1}(M)^{G} \cong \mathbb{Z}^{k_{1}} \oplus T_{\pi}^{1} \oplus T_{\pi^{\prime}}^{1}$ and $H_{1}\left(M_{G}\right) \cong \mathbb{Z}^{k_{2}} \oplus T_{\pi}^{2} \oplus T_{\pi^{\prime}}^{2}$, where $k_{i}, i=1,2$, are the first Betti numbers, while $T_{\pi}^{i} \oplus T_{\pi^{\prime}}^{i}, i=1,2$, are the torsion parts. Then $T_{\pi^{\prime}}^{1} \cong T_{\pi^{\prime}}^{2}$ and $k_{1}=k_{2}$.

Proof. It follows from a transfer argument [2, page 119, 2.2] that there exist two homomorphisms $\pi_{*}: H_{1}(M)^{G} \longrightarrow H_{1}\left(M_{G}\right)$ and $\mu_{*}: H_{1}\left(M_{G}\right) \longrightarrow$ $H_{1}(M)^{G}$ such that

$$
\begin{aligned}
& \pi_{*} \mu_{*}=|G|: H_{1}\left(M_{G}\right) \longrightarrow H_{1}\left(M_{G}\right) \\
& \mu_{*} \pi_{*}=|G|: H_{1}(M)^{G} \longrightarrow H_{1}(M)^{G}
\end{aligned}
$$

where $|G|$ stands for multiplication times $|G|$. Lemma 1 follows from the remark that the restriction of $|G|$ to $T_{\pi^{\prime}}^{1}$ and $T_{\pi^{\prime}}^{2}$ is an isomorphism because $|G|$ and $\left|T_{\pi^{\prime}}^{1}\right|$ respectively $\left|T_{\pi^{\prime}}^{2}\right|$ are relatively prime. The same kind of argument proves that $k_{1}=k_{2}$.

Proof of Theorem 1. Let us first suppose that $G$ is a $p$-group. Assume that $G$ acts homologically trivially. We have the following short exact sequence of groups

$$
1 \longrightarrow \pi_{1}(M) \longrightarrow \pi_{1}(M / G) \longrightarrow G \longrightarrow 1 .
$$

Since $G$ is abelian we have that $\pi_{1}(M)$ contains the first commutator subgroup of $\pi_{1}(M / G)$. In particular the above sequence induces the following

$$
1 \longrightarrow \pi_{1}(M) / \pi_{1}(M)^{(1)} \longrightarrow \pi_{1}(M / G) / \pi_{1}(M)^{(1)} \longrightarrow G \longrightarrow 1
$$

where $\pi_{1}(M)^{(1)}$ is the first commutator subgroup of $\pi_{1}(M)$ so that $\pi_{1}(M) /$ $\pi_{1}(M)^{(1)}=H_{1}(M)$. Moreover $\pi_{1}(M)^{(1)}$ is normal in $\pi_{1}(M / G)$ so the quotient makes sense. We thus see that $\pi_{1}(M / G) / \pi_{1}(M)^{(1)}$ is the extension of an abelian group by a cyclic group acting trivially on it. Since any element of this extension can be written as a product of an element in the normal abelian subgroup times a power of a counterimage of a generator of the cyclic group, then $[\mathbf{3 0}$, page $17,2.26]$ the extension is abelian. This implies that the first commutator subgroup $\pi_{1}(M / G)^{(1)}$ of $\pi_{1}(M / G)$ is contained in $\pi_{1}(M)^{(1)}$. But it is also true that $\pi_{1}(M)^{(1)} \subset \pi_{1}(M / G)^{(1)}$ and we conclude that $\pi_{1}(M)^{(1)} \cong \pi_{1}(M / G)^{(1)}$, that is, $\pi_{1}(M / G) / \pi_{1}(M)^{(1)}=H_{1}(M / G)$. From the last short exact sequence above we conclude that if $G$ is a $p$-group acting homologically trivially on $M$, then $H_{1}(M / G)$ is an extension of a normal subgroup $H \cong H_{1}(M)$ by a cyclic subgroup $F \cong G$.

Suppose now that $G$ is a cyclic group. If $G$ acts homologically trivially on $M$, then all its $p$-Sylow subgroups (which are cyclic) act homologically trivially on $M$, since $G$ is the direct product of its $p$-Sylow subgroups. Consider the sequence of coverings of $M / G$ associated to the $p$-Sylow subgroups 
of $G$. All we need to show is that the $p$-Sylow subgroups must act homologically trivially on their associated covering and conclude by an inductive argument. Let $|G|=p_{1}^{r_{1}} p_{2}^{r_{2}} \cdots p_{k}^{r_{k}}$, with $p_{1}<p_{2}<\cdots<p_{k}$. Let $M_{0}:=M$ and $M_{i}:=M_{i-1} / \mathbb{Z}_{p_{i}^{r_{i}}}, i=1, \ldots, k$. The Theorem is proved for the homologically trivial action of $\mathbb{Z}_{p_{1}^{r_{1}}}$ over $M_{0}$. Then we have that $H_{1}\left(M_{1}\right)$ is an extension of $H_{1}\left(M_{0}\right)$ by $\mathbb{Z}_{p_{1}^{r_{1}}}$. Now the group $\mathbb{Z}_{p_{2}}^{r_{2}}$ acts trivially on the normal subgroup $H_{1}\left(M_{0}\right)$. But it must also act trivially on $\mathbb{Z}_{p_{1}^{r_{1}}}$ since the order of the group of its automorphisms is $p_{1}^{r_{1}-1}\left(p_{1}-1\right)$.

This finishes the proof of the first part of Theorem 1 .

The reverse implication is actually trivial.

This finishes the proof.

Theorem 1 characterizes $H_{1}\left(M_{G}\right)$ only in the case that $G$ acts freely on $M$, because in this case $H_{1}\left(M_{G}\right)=H_{1}(M / G)$ (see above). On the other side, if $G$ acts on $M$ with non-empty fixed-point set, then $H_{1}\left(M_{G}\right)$ and $H_{1}(M / G)$ are different, in general. Howewer we can prove the following (it is essential that the dimension of the manifold is 3$)$ :

Theorem 2. Let $M$ be a closed 3-manifold and $G$ a finite cyclic group of prime order $p$ acting smoothly and orientation-preservingly on $M$. Suppose that $G$ acts with non-empty fixed-point set and that $H_{1}(M)$ has finite order prime with $p$. Then $G$ acts homologically trivially on $M$ if and only if

$$
H_{1}\left(M_{G}\right) \cong H_{1}(M) \text {. }
$$

Lemma 2. Let $M$ be a closed 3-manifold, $G$ a finite cyclic group of prime order $p$ acting smoothly and orientation-preservingly on $M$. Suppose that $H_{1}(M)$ has finite order and that its order is prime with $p$. If $G$ acts on $M$ with non-empty fixed-point set, then

$$
H_{1}\left(M_{G} ; \mathbb{Z}_{p}\right) \cong\{0\} .
$$

Remark. We shall be able to extend Lemma 2 to the case that $p$ divides the order of $H_{1}(M)$ in Section 4 by considering a specific 3-dimensional situation.

Proof. By hypothesis the order of $H_{1}(M)$ is finite and prime with $p$. Hence $M$ is a $\mathbb{Z}_{p}$-homology 3 -sphere. It follows from classical Smith's theory [2, Theorem 5.1, page 129] that $\operatorname{Fix}(G)$ is a $\mathbb{Z}_{p}$-homology sphere. Since $G$ acts on $M$ orientation-preservingly, then $\operatorname{Fix}(G)$ has dimension 1 and we conclude that $\operatorname{Fix}(G) \cong \mathbf{S}^{1}$. Consider a toral neighbourhood $U$ of $\operatorname{Fix}(G)$ in $M$ invariant by the $G$-action. Let $M^{\prime}$ be the closed complement of $U$. The manifold $M_{G}$ is obtained by glueing a solid torus to $M_{G}^{\prime}:=M^{\prime} / G$ (observe that $G$ acts freely on $\left.M^{\prime}\right)$. It follows from Lemma 1 that $H_{1}\left(M_{G}\right)$ is finite. Consider the following diagram in homology 


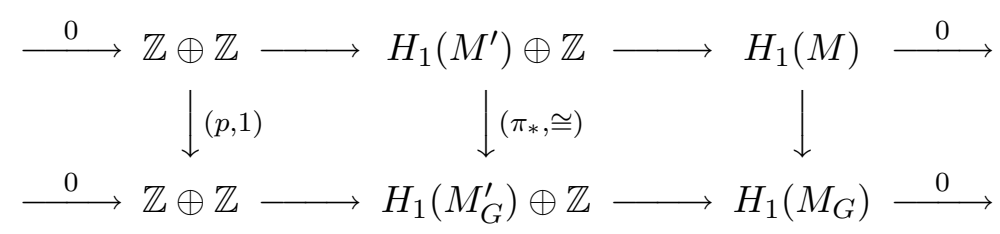

where $\mathbb{Z} \oplus \mathbb{Z} \cong H_{1}(\partial U), \mathbb{Z} \cong H_{1}(U)$ and the vertical maps are induced by projection. Notice that the 0 horizontal maps are due to the fact that the groups $H_{1}(M)$ and $H_{1}\left(M_{G}\right)$ are finite; moreover, considering the usual short exact sequence of fundamental groups and the abelianizing, it is easy to convince oneself that the image of the map $\pi_{*}$ has index $p$ in $H_{1}\left(M_{G}^{\prime}\right)$. Since, by hypothesis, $H_{1}(M)$ has no $p$-torsion, a diagram-chase argument shows that $H_{1}\left(M_{G}\right)$ cannot have $p$-torsion as well. Remark that the important fact here is that the first vertical map is multiplication times $p$ on the first factor, i.e. a standard meridian winds up $p$ times along itself.

This finishes the proof of Lemma 2.

Proof of Theorem 2. This is now an easy consequence of Lemmata 1 and 2.

\section{Cyclic coverings of knots.}

In this Section we specialize to the case of a 3 -manifold $M$ which is a cyclic covering of a knot $K$ in $\mathbf{S}^{3}$.

We first give an example of a homologically trivial action on the 2-fold covering of a hyperbolic knot, which is a direct application of the results of Section 3 and the Dehn surgery construction we have described in Section 2 .

Let $K \subset \mathbf{S}^{3}$ be an invertible hyperbolic knot with orientation-preserving symmetry group $\mathbf{D}_{n}$ for $n \geq 2$ (such examples for $n=2,4$ can be found already in the class of 2-bridge knots [4]). In particular $K$ admits at least two distinct inversions, say $t_{1}$ and $t_{2}$. As we have seen in Section 2 , for all but finitely many surgery coefficients, the manifold $M$ obtained by Dehn surgery on $K$ is hyperbolic and $t_{i}(i=1,2)$ extends to an involution $\bar{t}_{i}$ of $M$. We can also assume that $M$ is not a homology 3 -sphere. It is known [19] that the quotient $M / \bar{t}_{i}$ is a 3-orbifold with underlying topological space $\mathbf{S}^{3}$ and singular set a link with singular index 2 . It follows from Lemma 1 that $\bar{t}_{i}$ acts fixed-point freely on $H_{1}(M)$. But there exists a unique fixedpoint free action of $\mathbb{Z}_{2}$ on a finite abelian group, sending each element to its inverse. Thus $\bar{t}_{1}$ and $\bar{t}_{2}$ induce the same action on $H_{1}(M)$ and their product $\bar{t}_{1} \bar{t}_{2}$ acts homologically trivially on $M$.

More generally, if a hyperbolic 3-manifold $M$ is the 2-fold branched covering of two inequivalent knots, then there are two distinct isometries of $M$ 
which induce the same action on $H_{1}(M)$ and their product acts homologically trivially on $M$ (an example of a hyperbolic 3-manifold which is the 2 -fold branched covering of 4 inequivalent knots can be found in [28]).

The case of $n$-fold cyclic coverings of knots with $n>2$ is more complicated and it is subject of the rest of this section.

Let $M$ be a cyclic covering of a knot $K$ in $\mathbf{S}^{3}$. As we have explained in the Introduction, we first study only diffeomorphisms of $M$ which are not hidden to $K$, that is they descend to diffeomorphisms of $K$. So the question we are concerned with is the following: Let $g$ be a diffeomorphism of $M$ of finite order descending to a diffeomorphism of $K$. Give necessary and sufficient conditions for $g$ to act homologically trivially on $M$.

We distinguish two possible cases according if $g$ descends to a diffeomorphism of $K$ which acts freely or not. Accordingly, this section is divided into two parts. In the first part we show that the free case can always be reduced to the fixed-point one. In the second part we show that if $g$ descends to a diffeomorphism of $K$ with non-empty fixed-point set, then $g$ acts homologically trivially on $M$ if and only if either $K$ satisfies the $\mathcal{M}$-condition or the $\mathcal{M M}$-condition. The main theorem of this section is Theorem 4.

i) Free case.

The case of free actions is reduced to the case of actions with fixed-points by the following:

Theorem 3. Let $M$ be a p-fold cyclic branched covering of a knot $K$ in $\mathbf{S}^{3}$ and $\tilde{g}$ an orientation-preserving finite order diffeomorphism of $M$ which descends to a free diffeomorphism of $\left(\mathbf{S}^{3}, K\right)$. If $\tilde{g}$ acts homologically trivially on $M$, then there exists a 3-manifold $M^{\prime}$ obtained by Dehn surgery on $M$ which is a p-fold cyclic branched covering of a knot $K^{\prime}$ in an integral homology 3-sphere such that $\tilde{g}$ extends to a finite order diffeomorphism $\tilde{g}^{\prime}$ of $M^{\prime}$ acting homologically trivially on $M^{\prime}$ with non-empty fixed-point set.

Proof. The construction which follows is a minor application of a method due to Hartley [13].

Let $g$ be the diffeomorphism of $\left(\mathbf{S}^{3}, K\right)$ which is covered by $\tilde{g}$. Let $T \subset \mathbf{S}^{3}$ be a knot such that $T \cap K=\emptyset$ and $T$ is invariant under $g$. Denote by $G$ the group generated by $g$, by $Q$ the quotient manifold $\mathbf{S}^{3} / G$, by $\bar{T}$ the projection of $T$, by $\bar{K}$ the projection of $K$. Since $G$ acts freely and is cyclic (see Theorem 1) we have that $H_{1}(Q) \cong G$. As a cycle the projection $\bar{T}$ of $T$ represents a generator of $H_{1}(Q)$, otherwise $T$ would have more than one component. Denote by $Y$ the closed complement in $Q$ of a tubular neighbourhood $N(\bar{T})$ of $\bar{T}$ such that $\partial N(\bar{T}) \cap \bar{K}=\emptyset$. By [13, page 92] $H_{1}(Y) \cong \mathbb{Z}$ and the generator of $H_{1}(Y)$ may be represented by a curve in $\partial N(\bar{T})$. Thus there exist infinitely many surgery coefficients on $\bar{T}$ such that 
the surgered manifold is a homology 3 -sphere. Denote by $Q^{\prime}$ a homology $3-$ sphere obtained by Dehn surgery on $\bar{T} \subset Q$, by $\bar{T}^{\prime}$ the core of the added solid torus and by $\bar{K}^{\prime}$ the image of $\bar{K}$ in $Q^{\prime}$. In particular $Q^{\prime}-\bar{T}^{\prime}$ is homeomorphic to $Q-\bar{T}$. Let $\Sigma$ be the $G$-fold cyclic covering of $Q^{\prime}$ branched over $\bar{T}^{\prime}$. Then $\Sigma[\mathbf{1 3}$, page 92$]$ is a homology 3 -sphere obtained by Dehn surgery on $T \subset \mathbf{S}^{3}$ and the diffeomorphism $g$ extends to a diffeomorphism $g^{\prime}$ of $\Sigma$ fixing pointwise the preimage $T^{\prime}$ of $\bar{T}^{\prime}$. Denote by $K^{\prime}$ the image of $K$ in $\Sigma$. The $p$-fold cyclic branched covering $M^{\prime}$ of $\Sigma$ branched along $K^{\prime}$ is obtained by equivariant Dehn surgery of $M$ on the preimage (possibly disconnected) $\tilde{T}$ of $T$ in $M$. Finally the diffeomorphism $g^{\prime}$ lifts to a diffeomorphism $\tilde{g}^{\prime}$ of $M^{\prime}$ with fixed-point set the preimage $\tilde{T}^{\prime}$ of $T^{\prime}$ in $M^{\prime}$.

Up to now we have proved that there exists a 3 -manifold $M^{\prime}$ obtained by Dehn surgery on $M$ which is a $p$-fold cyclic branched covering of a knot $K^{\prime}$ in a integral homology 3 -sphere such that $\tilde{g}$ extends to a diffeomorphism $\tilde{g}^{\prime}$ of $M^{\prime}$. We now show that if $\tilde{g}$ acts homologically trivially on $M$, then $\tilde{g}^{\prime}$ acts homologically trivially on $M^{\prime}$.

To fix ideas, suppose that $\tilde{T}$ has $r$ components. By [14] $H_{1}(M) \oplus \mathbb{Z}^{r} \cong$ $H_{1}(M-\tilde{T})$ where the second factor $\mathbb{Z}^{r}$ is generated by some meridianal curves in the boundary $\partial N(\tilde{T})$ of an equivariant regular neighbourhood $N(\tilde{T})$ of $\tilde{T}$. Since $\tilde{g}$ is orientation-preserving, then it acts homologically trivially on $\partial N$. By hypothesis we know that it acts homologically trivially on $M$; therefore it acts trivially on $H_{1}(M-\tilde{T})$. Again for $M^{\prime}$ we have $H_{1}\left(M^{\prime}\right) \oplus \mathbb{Z}^{r} \cong H_{1}\left(M^{\prime}-\tilde{T}^{\prime}\right) \cong H_{1}(M-\tilde{T})$ and the action of $\tilde{g}^{\prime}$ on $H_{1}\left(M^{\prime}-\tilde{T}^{\prime}\right)$ coincides with the action of $\tilde{g}$ on $H_{1}(M-\tilde{T})$. Thus $\tilde{g}^{\prime}$ acts homologically trivially on $M^{\prime}$. Note that the roles of $M$ and $M^{\prime}$ can be interchanged so that $\tilde{g}$ acts homologically trivially if and only if $\tilde{g}^{\prime}$ does.

This finishes the proof.

Remark. If $M$ is hyperbolic, then $M^{\prime}$ in the statement of Theorem above can be chosen to be hyperbolic. This follows by applying Thurston's Hyperbolic Dehn Surgery Theorem to the construction. In fact we know that (see the notation above) there exists infinitely many surgery coefficients on $\bar{T}$ in $Q$ such that the surgered manifold $Q^{\prime}$ is a homology 3-sphere. If we suppose that $Q-\bar{T}$ is hyperbolic, by Thurston's Hyperbolic Dehn surgery Theorem, the resulting surgered manifold $Q^{\prime}$ is hyperbolic except for finitely many surgery coefficients.

\section{ii) Fixed-point case.}

We want to prove the following:

Theorem 4. Let $M$ be the $p$-fold cyclic branched covering of a knot $K$ in $\mathbf{S}^{3}$ which has cyclic period $q$ for some primes $p$ and $q, p$ odd. Denote by $G$ 
the cyclic group of diffeomorphisms of $\left(\mathbf{S}^{3}, K\right)$ of order $q$, by $K^{\prime}$ its fixedpoint set, by $E$ the group of diffeomorphisms of $M$ generated by the lifts of $G$. Suppose that:

i) $M$ is hyperbolic and not a homology 3-sphere.

ii) The linking number $l k\left(K, K^{\prime}\right)$ is 1 .

\section{Then:}

- Case: $p \neq q$; the group $E$ acts faithfully on $H_{1}(M)$ if and only if $K$ does not satisfy the $\mathcal{M}$-condition for the number $p$;

- Case: $p=q$; the group $E$ acts faithfully on $H_{1}(M)$ if and only if $K$ satisfies neither the $\mathcal{M}$-condition nor the $\mathcal{M M}$-condition.

The hypothesis in i) that $M$ is hyperbolic is not essential for the proof of Theorem 4. This hypothesis is requested only to guarantee that $E$ is a finite group.

Proof of Theorem 4. Denote by $C$ the cyclic group of covering transformations of the covering $M \longrightarrow\left(\mathbf{S}^{3}, K\right)$. The group $C \cong \mathbb{Z}_{p}$ fixes pointwise the preimage $\tilde{K}$ of $K$ in $M$ and acts locally as a group of rotations around $\tilde{K}$. On the other side, since $\operatorname{lk}\left(K, K^{\prime}\right)=1$ by hypothesis, the preimage $\tilde{K}^{\prime}$ of $K^{\prime}$ in $M$ is connected. Given a generator $g$ of $G$, there exists a lift $\tilde{g}$ of $g$ to $M$ which fixes pointwise $\tilde{K}^{\prime}$. Moreover $\tilde{g}$ fixes setwise the fixed-point set $\tilde{K}$ of $C$ and its restriction to $\tilde{K}$ is a rotation of order $q$. Thus $\tilde{g}$ and $C$ commute and we have a direct sum $E \cong C \oplus \tilde{G}$ where $\tilde{G} \cong G$ acts on $M$ with fixed-point set $\tilde{K}^{\prime}$. We shall denote by $M_{\tilde{G}}$ and $M_{E}$ the topological spaces underlying $M / \tilde{G}$ and $M / E$. By the positive solution of the Smith's conjecture, $K^{\prime}$ is unknotted and the action of $G$ on $\mathbf{S}^{3}$ is equivalent to a rotation around $K^{\prime}$. We conclude that $M_{E} \cong \mathbf{S}^{3}$ and that $M_{\tilde{G}}$ is the $p$-fold cyclic branched covering of $\mathbf{S}^{3}$ along the projection $\tilde{K}^{\prime}$ of $K^{\prime}$ to $M_{E}$.

a) The group $C$ does not act homologically trivially on $M$.

It follows from Theorem 1 that the cyclic group $C$ of covering transformations acts homologically trivially on $M$ if and only if $H_{1}(M / C)$ is a $p$-extension of $H_{1}(M)$. But $M / C$ is a 3 -orbifold with underlying topological space $\mathbf{S}^{3}$ and singular set the knot $K$ with singular index $p$. Therefore $H_{1}(M / C) \cong \mathbb{Z}_{p}$ and, since we know by hypothesis that $M$ is not a homology 3 -sphere, we conclude that the group $C$ does not act homologically trivially on $M$.

b) If $\tilde{G}$ acts homologically trivially on $M$, then $H_{1}(M / \tilde{G})$ is an extension of a normal subgroup $H \cong H_{1}(M)$ by a cyclic subgroup $F \cong \tilde{G}$.

This is simply Theorem 1.

c) The group $H_{1}(M / \tilde{G})$ maps onto $H_{1}\left(M_{\tilde{G}}\right)$ with kernel of order either $q$ or 1 . 
The group $\tilde{G}$ acts locally as a group of rotations around $\tilde{K}^{\prime}$. Let $U$ be a tubular neighbourhood of $\tilde{K}^{\prime}$ which is invariant under the action of $\tilde{G}$. Denote by $M^{\prime}$ the closed complement of $U$. The orbifold $M / \tilde{G}$ is obtained by glueing to $M_{\tilde{G}}^{\prime}:=M^{\prime} / \tilde{G}$ along the boundary a 3-orbifold with underlying topological space a solid torus and singular set the core of the torus with singular order $q$. The 3 -manifold $M_{\tilde{G}}$ is obtained by glueing to $M_{\tilde{G}}^{\prime}$ a solid torus. As a consequence of Seifert-Van Kampen theorem for fundamental groups, the group $H_{1}\left(M_{\tilde{G}}\right)$ is obtained from $H_{1}\left(M_{\tilde{G}}^{\prime}\right)$ by adding a relation $x=1$ for some $x \in H_{1}\left(M_{\tilde{G}}^{\prime}\right)$. On the other side the group $H_{1}(M / \tilde{G})$ is obtained from $H_{1}\left(M_{\tilde{G}}^{\prime}\right)$ by adding a relation $x^{q}=1$. More precisely

$$
\pi_{1}(M / \tilde{G}) \cong \pi_{1}\left(M_{\tilde{G}}^{\prime}\right) /\left\langle x^{q}\right\rangle \longrightarrow \pi_{1}\left(M_{\tilde{G}}\right) \cong \pi_{1}\left(M_{\tilde{G}}^{\prime}\right) /\langle x\rangle \longrightarrow 1
$$

and abelianizing

$$
H_{1}(M / \tilde{G}) \longrightarrow H_{1}\left(M_{\tilde{G}}\right) \longrightarrow 1 .
$$

Since we are quotienting out one $q$-torsion element at the most in an abelian group, it follows that $H_{1}(M / \tilde{G})$ surjects onto $H_{1}\left(M_{\tilde{G}}\right)$ with kernel of order either $q$ or 1 .

d) If $\tilde{G}$ acts homologically trivially on $M$, then $H_{1}\left(M_{\tilde{G}}\right) \cong H_{1}(M)$.

It follows from the discussion preceding a) that both $M$ and $M_{\tilde{G}}$ are $p$ fold cyclic branched coverings of knots in $\mathbf{S}^{3}$. Since $p$ is an odd prime, we can apply Plans's Theorem [24] to $M$ and $M_{\tilde{G}}$. Thus both $H_{1}(M)$ and $H_{1}\left(M_{\tilde{G}}\right)$ are direct doubles. Assume that $\tilde{G}$ acts homologically trivially on $M$. Because of Lemma 1 , it is enough to prove that $H_{1}(M)$ and $H_{1}\left(M_{\tilde{G}}\right)$ have isomorphic $q$-torsion parts. Since they are both direct doubles such torsion parts must be of the form:

$$
\bigoplus_{i=1}^{k}\left(\mathbb{Z}_{q^{r_{i}}} \oplus \mathbb{Z}_{q^{r_{i}}}\right) .
$$

From b) we deduce that the $q$-torsion part of $H_{1}(M)$ has index $q$ in the $q$ torsion part of $H_{1}(M / \tilde{G})$ which in its turn by c) surjects onto the $q$-torsion part of $H_{1}\left(M_{\tilde{G}}\right)$ with kernel of index $q$ (or 1 ). Since both $q$-torsion parts we are studying must be of the form $(*)$, we deduce that they are isomorphic.

e) If $H_{1}\left(M_{\tilde{G}}\right) \cong H_{1}(M)$, then $\tilde{G}$ acts homologically trivially on $M$.

Because of Lemma 1, it is sufficient to show that the action of $\tilde{G}$ on the $q$-torsion part of $H_{1}(M)$ is homologically trivial. Decompose the $q$-torsion part of $H_{1}(M)$ and $H_{1}(M)^{\tilde{G}}$ (see Lemma 1 for this notation) as a direct sum of cyclic groups. By the proof of Lemma 1 (the transfer argument) and since, by hypothesis, $H_{1}(M) \cong H_{1}\left(M_{\tilde{G}}\right)$, we see that the cyclic subgroups in the decomposition for $H_{1}(M)^{\tilde{G}}$ must have index 1 or $q$ in the cyclic subgroups 
of the decomposition for $H_{1}(M)$. But if an element of order $q$ acts trivially on a subgroup of index at most $q$, it must fix the whole group.

f) Case: $p \neq q$; the group $E$ acts faithfully on $H_{1}(M)$ if and only if $K$ does not satisfy the $\mathcal{M}$-condition for the number $p$.

If $p \neq q$, then $E$ is isomorphic to a cyclic group of order $p q$ and it acts faithfully on $H_{1}(M)$ if and only if both $C$ and $\tilde{G}$ do not act homologically trivially on $M$. It follows from a) and e) that $E$ acts faithfully on $H_{1}(M)$ if and only if $H_{1}(M) \cong H_{1}\left(M_{\tilde{G}}\right)$. By the proof of Lemma 1 this is true if and only if the order of $H_{1}(M)$ is equal to the order of $H_{1}\left(M_{\tilde{G}}\right)$. This is exactly the $\mathcal{M}$-condition for the knot $K$ and the number $p$.

g) Case: $p=q$; the group $E$ acts faithfully on $H_{1}(M)$ if and only if $K$ satisfies neither the $\mathcal{M}$-condition nor the $\mathcal{M M}$-condition for the number $p$.

In this case $E \cong C \oplus \tilde{G} \cong \mathbb{Z}_{p} \oplus \mathbb{Z}_{p}$ and any element of $E$ with non-empty fixed-point set lies either in $C$ or in $\tilde{G}$. We have seen in e) and f) that $\tilde{G}$ acts homologically trivially on $M$ if and only if $K$ satisfies the $\mathcal{M}$-condition for the number $p$. Suppose now that $\tilde{G}$ does not act homologically trivially on $M$. Then $E$ acts unfaithfully on $H_{1}(M)$ if and only if there is some finite subgroup $\tilde{F} \subset E$ projecting to $G$ and acting homologically trivially on $M$. Denote by $M / \tilde{F}$ the quotient orbifold and by $M_{\tilde{F}}$ its underlying topological space. As we have seen above $\tilde{F}$ has to act freely on $M$ and $M_{\tilde{F}}$ is a cyclic $p$-fold branched covering of the link $\bar{K} \cup \bar{K}^{\prime}$ in $M_{E} \cong \mathbf{S}^{3}$ ( $\bar{K}$ and $\bar{K}^{\prime}$ are the projections of $K$ and $K^{\prime}$ to $M_{E}$ ). By applying Theorem 1, we deduce that $F$ acts homologically trivially on $M$ if and only if the order of $H_{1}\left(M_{\tilde{F}}\right)$ is $p$ times the order of $H_{1}(M)$. This is exactly the $\mathcal{M M}$-condition (Section 2) for $K$ and the number $p$.

This finishes the proof of Theorem 4 .

\section{Proof of Theorem 5 .}

If the order $p$ of the covering is sufficiently large, as a consequence of Thurston's theory, then the isometry group of $M$ fixes setwise the preimage $\tilde{K}$ of $K$ and descends to the symmetry group of $K$. In fact the cyclic group $C$ of covering transformations acts locally as a group of rotations around its fixed-point set which is the preimage $\tilde{K}$ of $K$ in $M$. It follows from Thurston's theory $([\mathbf{3 1}]$ and $[\mathbf{1 7}])$ that $\tilde{K}$ is the unique shortest geodesic of $M$. Thus any isometry of $M$ fixes setwise $\tilde{K}$ and normalizes $C$ in the isometry group of $M$. Since, by hypothesis, $K$ has no cyclic or free periods, the orientation-preserving symmetry group of $K$ is trivial or isomorphic to $\mathbb{Z}_{2}$ according if $K$ is not invertible or it is. Thus the orientation-preserving isometry group $\mathrm{IsO}_{+}(M)$ of $M$ either coincides with the cyclic group $\mathbb{Z}_{p}$ of covering transformations or it is a dihedral group $\mathbf{D}_{p}$ of order $2 p$. In the first 
case it follows from Theorem 1 that the cyclic group $C$ of covering transformations acts homologically trivially on $M$ if and only if $H_{1}(M / C)$ is a $p$-extension of $H_{1}(M)$. But $M / C$ is a 3-orbifold with underlying topological space $\mathbf{S}^{3}$ and singular set the knot $K$ with singular index $p$. Therefore $H_{1}(M / C) \cong \mathbb{Z}_{p}$ and, since we know by hypothesis that $M$ is not a homology 3 -sphere, we conclude that $\operatorname{Iso}_{+}(M) \cong C$ acts faithfully on $H_{1}(M)$. In the second case let $N$ be the subgroup of $\mathrm{IsO}_{+}(M)$ acting homologically trivially on $M$. By the first part of the proof we know that $C \cap N=\{0\}$; thus $N$ is a proper normal subgroup of $\operatorname{Iso}_{+}(M)$. But $\operatorname{Iso}_{+}(M) \cong \mathbf{D}_{p}$ and this implies that $N$ is trivial.

\section{Proof of Theorem 6.}

By repeating the same argument as in the proof of Theorem 5, we know that neither the cyclic group of covering transformations of $M$ nor any isometry of $M$ which descends to an inversion of $K$ act homologically trivially on $M$. Thus $\mathrm{Iso}_{+}(M)$ acts faithfully on $M$ if and only if no isometry of prime order descending to a cyclic symmetry of $K$ acts homologically trivially on $M$. By taking $p$ greater than $\max \left(q_{1}, \ldots, q_{s}\right)$ the Theorem follows now from Theorem 4.

\section{Examples.}

In this section we will give some examples of periodic knots which satisfy the $\mathcal{M}$-condition.

Periodic knots which satisfy the $\mathcal{M}$-condition are rare and to construct them we rely on the following Theorem:

Theorem 7 (Davis-Livingston). A knot polynomial $\Delta$ which is congruent to 1 modulo $n$ is the Alexander polynomial of a knot of cyclic period $n$.

The proof of this Theorem can be found in [8, Corollary 1.2]. In this paper the authors investigate the following question: Given a knot polynomial $\Delta$ and an integer $n$, find necessary and sufficient conditions on $\Delta$ for the existence of a knot of cyclic period $n$ with Alexander polynomial $\Delta$. They are able to give a complete answer to this question in the case that the linking number between the knot and the fixed-point set of its cyclic periodic transformation is 1 (compare with the hypotheses of Theorem 4 and 6). In particular, given a knot polynomial congruent to 1 modulo $n$, Davis and Livingston construct explicitly a knot $K$ of cyclic period $n$ with the properties which we now describe. We repeat some notation from Section 2. If $K$ is a knot with cyclic period $n$ and $g$ a cyclic diffeomorphism of $\left(\mathbf{S}^{3}, K\right)$ with order $n$ with fixed-point set $K^{\prime}$, we have seen above that the topological space underlying the quotient $\mathbf{S}^{3} / G$, where $G$ is the group generated by $g$, is homeomorphic to $\mathbf{S}^{3}$; let $\bar{K}$ be the projection of $K$ to this quotient. Denote 
by $\Delta$ and $\bar{\Delta}$ the Alexander polynomials of $K$ respectively $\bar{K}$. Then Davis and Livingston prove that given a knot polynomial congruent to 1 modulo $n$, there exists a knot with cyclic period $n$ such that the linking number $\operatorname{lk}\left(K, K^{\prime}\right)$ is 1 and $\Delta=\bar{\Delta}$. Thus the orders of the first homology groups of the $p$-fold cyclic branched coverings $M$ and $\bar{M}$ of $K$, respectively $\bar{K}$, are equal and the $\mathcal{M}$-condition is automatically satisfied for these knots for the number $n$. There is no matter in reproducing here the drawings of [8] which are quite complicated. We have verified by the help of Weeks' SnapPea program that the easiest examples of [8] are hyperbolic.

\section{Hidden isometries.}

In this Section we want to discuss the problem of hidden isometries (see Section 1) for cyclic coverings of a knot and state some (partial) results in this direction.

Let $M$ be a hyperbolic $p$-fold cyclic branched covering of a knot $K$ in $\mathbf{S}^{3}$. Every symmetry of $K$ lifts to an isometry of $M$ but, in general, the full isometry group of $M$ contains the set of lifts of isometries of $K$ as a proper subgroup. We say that an isometry of $M$ is hidden to $K$ if it does not descend to a symmetry of $K$. The structure of the full isometry group of $M$ cannot be understood completely from $K$ in presence of hidden isometries (see [26] and [28] for a discussion of this topic). If the order $p$ of the covering is sufficiently large, as a consequence of Thurston's theory, there are no isometries of $M$ hidden to $K$. In fact the cyclic group $C$ of covering transformations acts locally as a group of rotations around its fixed-point set which is the preimage $\tilde{K}$ of $K$ in $M$. It follows from Thurston's theory that $\tilde{K}$ is the unique shortest geodesic of $M$. Thus any isometry of $M$ fixes setwise $\tilde{K}$ and normalizes $C$ in the isometry group of $M$. Therefore any isometry of $M$ descends to a symmetry of $K$ and, in this case, there are no hidden isometries. This motivates the hypotheses of Theorems 5 and 6 , namely taking the order of the covering sufficiently large. On the other side, for low order coverings, hidden isometries may occur $([\mathbf{2 8}])$.

About homologically trivial actions of hidden isometries, we have the following result:

Theorem 8. Let $M$ be the p-fold cyclic branched covering of a knot $K$ for some odd prime $p$. Let $g$ be an orientation-preserving isometry of prime odd order, say q. Suppose that:

i) $M$ is hyperbolic and not a homology 3-sphere.

ii) $H_{1}(M)$ has finite order.

iii) The order of $H_{1}(M)$ and $q$ are relatively prime.

If $g$ is hidden to $K$, then $g$ does not act homologically trivially on $M$. 
Proof. We argue by contradiction. We prove some intermediate steps a), b), c), d) under the hypothesis that $g$ acts homologically trivially on $M$ and we reach a contradiction in e).

Since $M$ is hyperbolic, the group $\operatorname{Iso}(M)$ of isometries of $M$ is finite. Denote by $\operatorname{Iso}_{+}(M)$ the orientation-preserving subgroup of $\operatorname{Iso}(M)$. Let $N$ be the subgroup of isometries of $M$ which act homologically trivially on $M$. The group $N$ is normal in $\operatorname{Iso}(M)$. Since $g$ acts homologically trivially on $M$, then the cyclic group $G \cong \mathbb{Z}_{q}$ generated by $g$ is a subgroup of $N$.

a) The Sylow q-subgroup of Iso+ $(M)$ is either cyclic or the direct sum of two cyclic groups.

Denote by $S_{q}$ the Sylow $q$-subgroup of $\mathrm{Iso}_{+}(M)$. Since $q$ is prime with the order of $H_{1}(M)$ by hypothesis, then $M$ is a $\mathbb{Z}_{q}$-homology 3 -sphere. If $S_{q}$ acts freely on $M$, it follows from [2, page 148, Theorem 8.1] and [3, page 157, Theorem 9.5] and the fact that $q$ is odd that $S_{q}$ is cyclic. Else, suppose that there is an isometry of order a power of $q$ with non-empty fixed-point set $\mathcal{F}$ and that $S_{q}$ is not cyclic. In this case we shall prove that $S_{q}$ is the direct sum of two cyclic groups (see [27] for a similar argument). By Smith's theory, the fixed-point set of an orientation-preserving isometry of prime odd order of $M$ is either empty or a $\mathbb{Z}_{q}$-homology 1 -sphere and in the latter case, the only possibility is that the fixed-point set is $\mathbf{S}^{1}$. Denote by $\bar{S}_{q}$ the maximal cyclic subgroup of $S_{q}$ fixing pointwise $\mathcal{F}$. By [30, page 88, 1.5] either $\bar{S}_{q}$ is normal in $S_{q}$ or there exists a subgroup $\bar{S}_{q}^{x}$ in the normalizer $N_{S_{q}}\left(\bar{S}_{q}\right)$ of $\bar{S}_{q}$ in $S_{q}$ conjugate to $\bar{S}_{q}$ and distinct from it.

Suppose first that there exists a subgroup $\bar{S}_{q}^{x}$ in the normalizer $N_{S_{q}}\left(\bar{S}_{q}\right)$ of $\bar{S}_{q}$ in $S_{q}$ conjugate to $\bar{S}_{q}$ and distinct from it. Because $\bar{S}_{q}^{x}$ is in the normalizer of $\bar{S}_{q}$ it fixes setwise the fixed-point set $\mathcal{F}$ of $\bar{S}_{q}$.

Remark 1. The orientation-preserving isometries which fix setwise a 1sphere either fix the curve pointwise, act as rotations on it or as reflections so the normalizer of a cyclic group fixing pointwise a 1-sphere is a subgroup in the semidirect product of $\mathbb{Z}_{2}$ (the reflection) and $\mathbb{Z}_{a} \oplus \mathbb{Z}_{b}$ (the isometries which fix the sphere pointwise and the rotations) for some positive integers $a$ and $b$ where $\mathbb{Z}_{2}$ acts on $\mathbb{Z}_{a} \oplus \mathbb{Z}_{b}$ by sending each element onto its inverse.

Since $q$ is odd and both $\bar{S}_{q}$ and $\bar{S}_{q}^{x}$ are subgroups of $N_{S_{q}}\left(\bar{S}_{q}\right)$, then we have an abelian subgroup $\bar{S}_{q} \oplus \bar{S}_{q}^{x}$. Moreover $\bar{S}_{q}^{x}$ fixes pointwise the curve $x(\mathcal{F})$ image of $\mathcal{F}$ and $x(\mathcal{F})$ is distinct from $\mathcal{F}$.

Now consider the group $\bar{S}_{q}^{x^{2}}$. The group $\bar{S}_{q}^{x} \oplus \bar{S}_{q}^{x^{2}}$ is conjugate to $\bar{S}_{q} \oplus \bar{S}_{q}^{x}$ and abelian. Both $\bar{S}_{q}$ and $\bar{S}_{q}^{x^{2}}$ fix setwise the fixed-point set $x(\mathcal{F})$ of $\bar{S}_{q}^{x}$ and Remark 1 implies that $\bar{S}_{q} \oplus \bar{S}_{q}^{x}=\bar{S}_{q}^{x} \oplus \bar{S}_{q}^{x^{2}}$.

Remark 2. Assume that there exist two distinct simple closed curves fixed pointwise by two distinct non-trivial elements in a direct double $\mathbb{Z}_{q} \oplus \mathbb{Z}_{q}$. 
In this case, if an element in the direct double has non-empty fixed-point set, then such fixed-point set coincides with one of these two curves by $[\mathbf{1}$, page 175 , Theorem 2.3].

Now $\bar{S}_{q}^{x^{2}}$ has non-empty fixed-point set $x^{2}(\mathcal{F})$ and, by Remark 2, either $x^{2}(\mathcal{F})=\mathcal{F}$ or $x^{2}(\mathcal{F})=x(\mathcal{F})$. If $x^{2}(\mathcal{F})=x(\mathcal{F})$, then $\bar{S}_{q}=\bar{S}_{q}^{x}$ which is impossible. Hence $x^{2}(\mathcal{F})=\mathcal{F}$ which implies $\bar{S}_{q}=\bar{S}_{q}^{x^{2}}$. Therefore $x^{2}$ belongs to the normalizer of $\left(\bar{S}_{q}\right)$ and since $x$ has odd order, we conclude that $x$ is in the normalizer of $\bar{S}_{p}$ which is a contradiction. So this case is impossible.

Therefore $\bar{S}_{q}$ is normal in $S_{q}$ and we conclude by applying Remark 1 .

b) The group $G$ acts freely on $M$. Moreover $G$ is normalized by the cyclic group $C \cong \mathbb{Z}_{q}$ of covering transformations of the covering $M \longrightarrow\left(\mathbf{S}^{3}, K\right)$.

Denote by $S_{q}^{\prime}$ the Sylow $q$-subgroup of $N$ containing $G$. The group $S_{q}^{\prime}$ is normalized by (a subgroup of Iso $(M)$ conjugate to) $C$ because all $q$-Sylow subgroups of $N$ are conjugate in $N$ and $N$ is normal. Suppose first that $G$ has non-empty fixed-point set $\mathcal{F}$. Since $g$ is orientation-preserving by hypothesis and $M$ a $\mathbb{Z}_{q}$-homology 3 -sphere, then $\mathcal{F} \cong \mathbf{S}^{1}$. According to the proof of a), if $S_{q}^{\prime}$ is the direct sum of two cyclic groups, since $p$ is odd $C$ must fix both cyclic groups. This means that $C$ normalizes the cyclic group containing $G$. Thus, in any case, if $g$ had non-empty fixed-point set, the elements of $C$ would leave it invariant acting as rotations (or the identity) on it. But this is impossible, because we have supposed that $g$ is hidden to $K$ and thus it does not commute with the elements of $C$. We conclude that $G$ acts freely on $M$ and, by the proof of a), that $S_{q}^{\prime}$ is cyclic. Moreover the subgroup $G$ of prime order $q$ is characteristic in $S_{q}^{\prime}$ and it is normalized by $C$.

c) Denote by $M / G$ the quotient manifold. Then $H_{1}(M / G) \cong H \oplus F$ where $H \cong H_{1}(M)$ and $F \cong G$. Moreover $C$ descends to a group of isometries $\bar{C}$ of $M / G$ and the induced action on $H_{1}(M / G)$ is fixed-point free.

It follows from Theorem 1 and b) that $H_{1}(M / G)$ is an extension of a normal subgroup $H \cong H_{1}(M)$ by a cyclic subgroup $F \cong G$. Moreover, since we know by hypothesis that the order $q$ of $G$ and the order of $H_{1}(M)$ are relatively prime, we conclude $[\mathbf{3 0}$, page $231,8.5]$ that $H_{1}(M / G)$ is a direct sum $H_{1}(M / G) \cong H \oplus F$.

This finishes the first part of the proof.

Denote by $E \subset \mathrm{Iso}_{+}(M)$ the group generated by $G$ and $C$. It follows from b) that the group $G$ is normal in $E$ and that $E$ splits as a semidirect product of $G$ and $C$. Since, by hypothesis, $G$ is hidden to $K$, then no nontrivial element of $G$ commutes with $C$, that is $C$ acts fixed-point freely by inner conjugation on $G-\{1\}$. The covering $M \longrightarrow M / E$ factors through 
the covering $M \longrightarrow M_{G}=M / G$ and the factor group $C=E / G$ descends to a cyclic group of isometries $\bar{C} \cong C$ of $M / G$ with non-empty fixed-point set. The group $\bar{C}$ acts on $H_{1}(M / G)$ and we claim that this action is fixedpoint free (apart from the identity element). Suppose, on the contrary, that $x \in H_{1}(M / G)$ is fixed by $\bar{C}$. If $x$ belonged to $H$, then $C$ would fix some non-trivial element of $H_{1}(M)$, which is impossible because $C$ cannot act homologically trivially on $M$. In fact, by Theorem $1, C$ acts homologically trivially on $M$ if and only if $M$ is an integral-homology 3-sphere, which is not by hypothesis. If $x$ were in $F$, then $C$ would fix some non-trivial element of $G$ which is also impossible because we have shown above that $C$ acts fixed-point freely (by conjugation) on $G$.

d) Denote by $E \subset \mathrm{IsO}_{+}(M)$ the group generated by $G$ and $C$, by $M / E$ the quotient orbifold and by $M_{E}$ the underlying topological space. Then $M_{E}$ is a homology 3-sphere.

It follows from c) that $\bar{C}$ is a $p$-group acting on $M / G$ and that the induced action on $H_{1}(M / G)$ is fixed-point free. Moreover $\bar{C}$ acts on $M / G$ with nonempty fixed-point set (this fixed-point set is the projection to $M / G$ of the fixed-point set of $C$ in $M)$. Finally note that the order of $H_{1}(M / G)$ is prime with $p$ because both the order of $H$ and the order of $F$ are (the order of $H$ is prime with $p$ by [12], the order of $F$ is prime with $p$ because we have proved in b) that the Sylow $q$-subgroup $S_{q}$ of $\operatorname{Iso}_{+}(M)$ is cyclic and $C$ does not commute with $C$ ). By applying Lemma 1 and the fact that $\bar{C}$ acts fixed-point freely on $H_{1}(M / G)=H_{1}\left(M_{G}\right)$, we conclude that $H_{1}\left(M_{E}\right)$ has only possibly got $p$-torsion. It follows now from Lemma 2 that $M_{E}$ is an integral homology 3 -sphere.

e) $G$ does not act homologically trivially on $M$.

If $G$ acts homologically trivially on $M$, then c) and d) above hold. Thus $M_{G}$ is a $p$-fold cyclic branched covering of a knot in the integral homology 3-sphere $M_{E}$. By Plans's Theorem [24] and the hypothesis that $q$ is odd, it follows that $H_{1}\left(M_{G}\right)$ is a direct double. But this contradicts c) because $H_{1}\left(M_{G}\right)=H_{1}(M / G) \cong H \oplus F$ and the order of $F \cong G$ is prime with the order of $H \cong H_{1}(M)$ by hypothesis.

This finishes the proof.

\section{References}

[1] A. Borel, Seminar on transformation groups, Ann. Math. Studies, 46, Princeton University Press, Princeton, 1960.

[2] G. Bredon, Introduction to compact transformation groups, Academic Press, New York, 1972.

[3] K. Brown, Cohomology of groups, Graduate Texts in Math., 87, Springer-Verlag, New York, 1982. 
[4] G. Burde and H. Zieschang, Knots, De Gruyter Studies in Math., 5, De Gruyter, Berlin, 1985.

[5] V. Chumillas and J.M. Montesinos, The homology of cyclic and irregular dihedral branched covers of $\mathbf{S}^{3}$, Math. Ann., 280 (1988), 483-500.

[6] J.F. Davis, The homology of cyclic branched covers of $\mathbf{S}^{3}$, Math. Ann., 301 (1995), 507-518.

[7] (1995), 519-528.

[8] J.F. Davis and C. Livingston, Alexander polynomials of periodic knots, Topology, 30(4) (1991), 551-564.

[9] P. Del Val and C. Weber, Plans Theorem for links, Top. and its Appl., 34 (1990), 247-255.

[10] R.H. Fox, A quick trip through knot theory, Topology of 3-manifolds and related topics, Proc. Univ. Georgia Inst., Prentice-Hall, New York, (1962), 120-167.

[11] C. McA. Gordon, Knots whose branched cyclic coverings have periodic homology, Trans. Amer. Math. Soc., 168 (1972), 357-370.

[12] - Some aspects of classical knot theory, Knot theory, Lect. Notes Math., 685, Springer, Plans-sur-Bex, 1977.

[13] R.I. Hartley, Knots with free period, Can. J. Math., 33 (1981), 91-102.

[14] R.I. Hartley and K. Murasugi, Homology invariants, Can. J. Math., 30 (1978), 655670.

[15] F. Hosokawa and S. Kinoshita, On the homology group of the branched cyclic covering spaces of links, Osaka Math. J., 12 (1960), 331-355.

[16] S. Kojima, Bounding finite groups acting on 3-manifolds, Math. Proc. Camb. Phil. Soc., 96 (1984), 269-281.

[17] _ Determining knots by branched covers, Low-dimensional topology and Kleinian groups, London Math. Soc. Lecture Notes, 112 (D.B.A. Epstein ed.), 193207.

[18] J.P .Mayberry and K. Murasugi, Torsion-groups of abelian coverings of links, Trans. Amer. Math. Soc., 271(1) (1982), 143-173.

[19] J.M. Montesinos, Surgery on links and double branched covers, Knots, groups and 3-manifolds, Ann. Math. Studies, 84, Princeton, (1975), 227-259.

[20] J. Morgan and H. Bass (eds.), The Smith Conjecture, Academic Press, New York, 1984.

[21] K. Murasugi, On periodic knots, Comment. Math. Helv., 46 (1971), 162-174.

[22] _ On symmetries of knots, Tsukuba J. Math., 4 (1980), 331-347.

[23] S. Naik, Periodicity, genera and Alexander polynomials of knots, Pacific J. Math., 166(2) (1994), 357-371.

[24] A. Plans, Aportación al estudio de los grupos de homologia de los recubrimientos ciclicos ramificados correspondientes a un nudo, Rev. Acad. Ci. Madrid, 47 (1953), 161-193.

[25] J.G. Ratcliffe, Foundations of Hyperbolic Manifolds, Graduate Texts in Math., 149, Springer-Verlag, New York, 1994. 
[26] M. Reni, On the order of the isometry groups of cyclic coverings of hyperbolic knots, Preprint, 1996.

[27] _ Finite group actions on homology 3-spheres, to appear in Mh. Math.

[28] M. Reni and B. Zimmermann, Isometry groups of hyperbolic 3-manifolds which are cyclic branched coverings, to appear in Geom. Dedicata.

[29] M. Sakuma, On the polynomials of periodic links, Math. Ann., 257 (1981), 487-494.

[30] M. Suzuki, Group Theory I, Grundlehren der Mathematischen Wissenschaften, 247, Springer-Verlag, Berlin, 1982.

[31] W. Thurston, The geometry and topology of three-dimensional manifolds, Lecture Notes, Princeton University Press, 1978.

Received May 30, 1997 and revised December 10, 1997.

\author{
SISSA-ISAS \\ 34014 TRIESTE \\ ITALY \\ E-mail address: paoluzzi@sissa.it \\ Università Degli Studi di TRIeste \\ Piazzale Europa, 1 \\ 34100 TRIESTE \\ ITALY \\ E-mail address: reni@univ.trieste.it
}

